\title{
All-trans retinoic acid suppresses apoptosis in PC12 cells injured by oxygen and glucose deprivation via the retinoic acid receptor $\alpha$ signaling pathway
}

\author{
XIAOJIAN ZHANG ${ }^{1,2}$, QIN YU ${ }^{1,2}$, WEI JIANG ${ }^{1,2}$, YANG BI $^{1-3}$, YUN ZHANG ${ }^{1,2}$, \\ MIN GONG ${ }^{1,2}$, XIAOPING WEI ${ }^{1,2}$, TINGYU LI ${ }^{1,2}$ and JIE CHEN ${ }^{1,2}$ \\ ${ }^{1}$ Children's Nutritional Research Center; ${ }^{2}$ Ministry of Education Key Laboratory of Child Development and Disorders, \\ Chongqing Key Laboratory of Translational Medical Research in Cognitive Development and Learning and Memory Disorders; \\ ${ }^{3}$ Stem Cell Biology and Therapy Laboratory, Children's Hospital of Chongqing Medical University, \\ Chongqing 400014, P.R. China
}

Received November 30, 2013; Accepted May 29, 2014

DOI: $10.3892 / \mathrm{mmr} .2014 .2568$

\begin{abstract}
Vitamin A (VA) has a number of important biological functions in human growth and development. Previous studies by our group demonstrated that the normal VA levels improved recovery of learning and memory function and decreased apoptosis in rats with hypoxic-ischemic brain damage (HIBD). However, it has not been fully elucidated how VA regulates the apoptosis of neuronal cells. To investigate the anti-apoptotic effect of VA, an in vitro oxygen glucose deprivation (OGD) model in PC12 cells was treated with four concentrations of all-trans-retinoic acid (ATRA), an active in vivo product of VA. Following in vitro OGD injury in PC12 cells, the percentage of apoptosis and the fluorescence intensity of the mitochondrial membrane potential (MMP) were increased in the cells, and the expression levels of B-cell lymphoma-associated X (Bax) were enhanced. ATRA treatment at $2-4 \mu \mathrm{mol} / 1$ for $24 \mathrm{~h}$ decreased the percentage of apoptosis and the MMP of the PC12 cells injured by OGD. ATRA at $4 \mu \mathrm{mol} / 1$ also reduced the expression levels of Bax and enhanced the expression of B-cell lymphoma 2. Furthermore, RNA interference with retinoic acid receptor $\alpha(\mathrm{RAR} \alpha)$ reversed the observed effect in PC12 cells following ATRA treatment at $4 \mu \mathrm{mol} / 1$ alone. In conclusion, the present study suggested that treatment with ATRA at $4 \mu \mathrm{mol} / \mathrm{l}$
\end{abstract}

Correspondence to: Dr Jie Chen, Children's Nutritional Research Center, Children's Hospital of Chongqing Medical University, 136 Zhongshan Er Road, Yuzhong, Chongqing 400014, P.R. China E-mail: jchen010@gmail.com

Abbreviations: HIBD, hypoxia-ischemia brain damage; VA, vitamin A; RA, retinoic acid; PC12 cells, rheochromocytoma cells; OGD, oxygen glucose deprivation; RFP, red fluorescent protein; RAR $\alpha$, retinoic acid receptor $\alpha$; MMP, mitochondrial membrane potential

Key words: all-trans-retinoic acid, PC12 cells, apoptosis, oxygen and glucose deprivation, retinoic acid receptor $\alpha$ suppressed apoptosis of PC12 cells following OGD injury, potentially through regulation of the RAR $\alpha$ signaling pathway.

\section{Introduction}

Vitamin A (VA) is an essential fat-soluble micronutrient. Retinoic acid (RA) is the main active metabolite of VA in vivo, and it exists in three isomers: All-trans-retinoic acid (ATRA), 13-cis-retinoic acid (13-cis-RA) and 9-cis-retinoic acid (9-cis-RA). ATRA is able to regulate the transcriptional activity of downstream target genes through retinoic acid receptor (RAR)-mediated signal transduction. RARs consist of two families, the RARs and the retinoid X receptors (RXRs), with each family composed of three isotypes: $\alpha, \beta$ and $\gamma$. The RAR $\alpha$ subtype has an important role in neuronal development (1-3).

A previous study by our group identified that neonatal rats with VA deficiency during the gestational period exhibited a reduction in the expression of RAR $\alpha$, coupled with deficits in learning and memory function in adulthood (4). It has also been demonstrated that a VA deficiency reduces the recovery ability of learning and memory function in pups following hypoxic-ischemic brain damage (HIBD), which is regulated by the RAR $\alpha$ signaling pathway (5). The RAR $\alpha$ signaling pathway is activated by ATRA, which triggers a variety of biological functions. Several previous studies investigated the mechanism of apoptosis in neuronal cells and the effect of antagonizing neuronal apoptosis in brain damage (6-14). However, whether VA has an anti-apoptotic function and the mechanisms underlying these effects in neuronal cells have remained elusive.

The present study aimed to establish an oxygen and glucose deprivation (OGD) model in vitro to investigate whether ATRA is neuroprotective by suppressing the apoptosis of neuronal cells. It was revealed that ATRA treatment at $4 \mu \mathrm{mol} / 1$ suppresses apoptosis of PC12 cells injured by OGD through the RAR $\alpha$ signaling pathway. The study of ATRA may facilitate and promote the clinical application of VA as an adjuvant therapy for patients with brain damage. 


\section{Materials and methods}

Culturing PC12 cells. PC12 cells were purchased from the Cell Bank of Shanghai Institute of Cell Biology, Chinese Academy of Sciences (Shanghai, China). The cells were cultured in Dulbecco's modified Eagle's medium (DMEM; Hyclone, Logan, UT, USA) containing 10\% horse serum (HS; Gibco-BRL, Grand Island, NY, USA), $\%$ fetal bovine serum (FBS; Gibco-BRL), 100 units $/ \mathrm{ml}$ penicillin and $100 \mu \mathrm{g} / \mathrm{ml}$ streptomycin at $37^{\circ} \mathrm{C}$ in a $5 \% \mathrm{CO}_{2}$ incubator. At $90 \%$ confluence, the $\mathrm{PC} 12$ cells were digested with TrypLE (Gibco-BRL) and passaged at a ratio of $1: 2$ every 3-4 days.

Construction of an in vitro OGD model. The PC12 cells were seeded and grown to $\sim 90 \%$ confluence in a six-well plate. To establish the OGD model, the culture medium of PC12 cells was changed to Earle's balanced salt solution (EBSS; Hyclone; $11.6 \mathrm{mmol} / 1 \mathrm{NaCl}, 5.4 \mathrm{mmol} / 1 \mathrm{KCl}, 0.8 \mathrm{mmol} / 1 \mathrm{MgSO}_{4}$, $10 \mathrm{mmol} / 1 \mathrm{NaH}_{2} \mathrm{PO}_{4}, 26.2 \mathrm{mmol} / 1 \mathrm{NaHCO}_{3}, 1.8 \mathrm{mmol} / 1 \mathrm{CaCl}_{2}$, $10 \mathrm{mg} / \mathrm{l}$ phenol red and no glucose; $\mathrm{pH}$ 7.4) following washing of the cells twice with D-Hank's solution, which was prepared by resolving Hank's balanced salt mixture (Hycyclone Laborotories, Inc., Omaha, Nebraska, USA) with double distilled water. The cells were then placed in an incubator (Thermo Forma 3111; Thermo Scientific; Waltham, MA, USA) with 5\% $\mathrm{O}_{2}$ and $95 \%$ $\mathrm{N}_{2}$ at $37^{\circ} \mathrm{C}$ for $6 \mathrm{~h}$.

ATRA treatment. ATRA (R2625; Sigma, St. Louis, MO, USA) was added to the culture medium (DMEM) of PC12 cells at final concentrations of $0.5,2,4$ and $20 \mu \mathrm{mol} / 1$ for $24 \mathrm{~h}$. Next, the PC12 cells pre-treated with each concentration of ATRA were immediately injured by OGD. PC12 cells without ATRA treatment served as a positive control.

RNA interference of RAR $\alpha$. A recombinant adenovirus of RAR $\alpha$-small interfering (si)RNA was designed and constructed in our laboratory (5). Three types of RAR $\alpha$-siRNA adenovirus were pooled to transfect into PC12 cells for $36 \mathrm{~h}$. A pair of unspecific siRNA was labeled with a red fluorescent protein, RFP, and served as a negative control. The infection efficiency and consistency of Ad-siRAR $\alpha$ and Ad-RFP were confirmed using fluorescent microscopy (Nijon TE2000-A; Nikon, Tokyo, Japan).

RNA isolation and quantitative polymerase chain reaction $(q P C R)$ analysis. Total RNA was isolated using an RNA extraction kit (Genemega Inc., San Diego, CA, USA) and reverse-transcribed into cDNA using the PrimeScript ${ }^{\circledR}$ RT reagent kit (DRR037A; Takara Bio, Inc., Shiga, Japan) using a Bio-Rad My Cycler (Bio-Rad Laboratories, Inc., Hercules, CA, USA) according to the manufacturer's instructions. The single-stranded cDNA was diluted 5- to 10-fold and used as the PCR template. The cDNA was quantified using real-time PCR with the StepOne v2.1 Real-Time PCR instrument (ABI; Redwood City, CA, USA) and RealMasterMix (SYBR Green; Tiangen Biotech Co., Ltd., Beijing, China). The procedure was performed as follows: Denaturation at $95^{\circ} \mathrm{C}$ for $10 \mathrm{~min}$, followed by 45 cycles at $95^{\circ} \mathrm{C}$ for $15 \mathrm{sec}, 60^{\circ} \mathrm{C}$ for $60 \mathrm{sec}$ and $72^{\circ} \mathrm{C}$ for $30 \mathrm{sec}$. Melting curve analysis and gel electrophoresis were utilized to ensure that a single PCR product was amplified in
Table I. Primer sequences for polymerase chain reaction.

\begin{tabular}{ll}
\hline Gene & \multicolumn{1}{c}{ Primer sequences } \\
\hline RAR $\alpha$ & F: 5'CAGGAGGGAGAAGGCAGTGAC3' \\
& R: 5'ATGGCTTGAGTTCGGAGGACAG3' \\
Bax & F: 5'AAGTAGAAGAGGGCAACCAC3' \\
& R: 5'GATGGCAACTTCAACTGGG3' \\
Bcl-2 & F: 5'CGGGAGAACAGGGTATGA3' \\
& R: 5'CAGGCTGGAAGGAGAAGAT3' \\
$\beta$-actin & F: 5'GCATAGCCACGCTTGTTCTTGAAG3' \\
& R: 5'GAACCGCTCATTGCCGATAGTG3'
\end{tabular}

RAR, retinoic acid receptor; Bcl-2, B-cell lymphoma 2; Bax, Bcl-2-associated X.

each reaction. The PCR primers were designed using Primer Premier 5.0 software (Premier Biosoft International, Paolo Alto, CA, USA). The primer sequences for RAR $\alpha$, B-cell lymphoma 2-associated X (Bax), B-cell lymphoma 2 (Bcl-2) and $\beta$-actin are summarized in Table I.

The data were presented as the $\mathrm{Ct}$ value and the ratio of the relative quantity of the target gene to $\beta$-actin was calculated. A semi-quantitative PCR reaction was conducted using the following protocol: $94^{\circ} \mathrm{C}$ for $20 \mathrm{sec}, 68^{\circ} \mathrm{C}$ for $30 \mathrm{sec}$ and $70^{\circ} \mathrm{C}$ for $20 \mathrm{sec}$ for 16 cycles, with a $1^{\circ} \mathrm{C}$ decrease per cycle, followed by $25-32$ cycles at $94^{\circ} \mathrm{C}$ for $20 \mathrm{sec}, 56^{\circ} \mathrm{C}$ for $30 \mathrm{sec}$ and $70^{\circ} \mathrm{C}$ for $20 \mathrm{sec}$. The PCR products were resolved on $1.5 \%$ agarose gels. All of the samples were normalized to the endogenous levels of $\beta$-actin. All operations were conducted in accordance with the standard protocol.

Western blot analysis. The cells were collected from dishes or wells and lysed in radioimmunoprecipitation assay (RIPA) buffer containing phenylmethanesulfonyl fluoride (Biotake Co., Ltd., Taiwan, China). The cell lysates were collected and the protein concentration was determined using a bicinchoninic acid protein concentration determination kit(BiotakeCo.,Ltd.,Beijing,China). The cell lysates were purified by $10 \%$ SDS-Page (Beyotime, China), with $\sim 20 \mu \mathrm{g}$ of total protein loaded per lane. Following electrophoresis, the proteins were transferred to an Immobilon-P membrane (Millipore, Billerica, MA, USA). Following blocking with 5\% FBS in Tris-buffered saline/Tween-20 (TBST) buffer at room temperature for $1 \mathrm{~h}$, the membrane was probed overnight with the primary antibodies, goat polyclonal anti-RAR $\alpha$ (28767; 1:200-1:2,000; Abcam, Cambridge, MA, USA), mouse monoclonal anti-Bax (B8429; 1:30-1:200; Sigma), mouse monoclonal anti-Bcl-2 (sc-7382; 1:100-1:1,000; Santa Cruz Biotechnology, Inc., Santa Cruz, CA, USA) and mouaw monoclonoal $\beta$-actin (sc-130065; 1:100-1:1,000; Santa Cruz Biotechnology, Inc.), respectively, at $4^{\circ} \mathrm{C}$ and was then incubated with the appropriate secondary antibody conjugated with horseradish peroxidase (Santa Cruz Biotechnology, Inc.) at room temperature for $1 \mathrm{~h}$. The protein was detected using an enhanced chemiluminescent substrate kit of chemiluminescent HRP substrate (Millipore, Billerica, MA, USA) and recorded using a Kodak 440CF Image Station (Kodak, Rochester, NY, USA). 
A
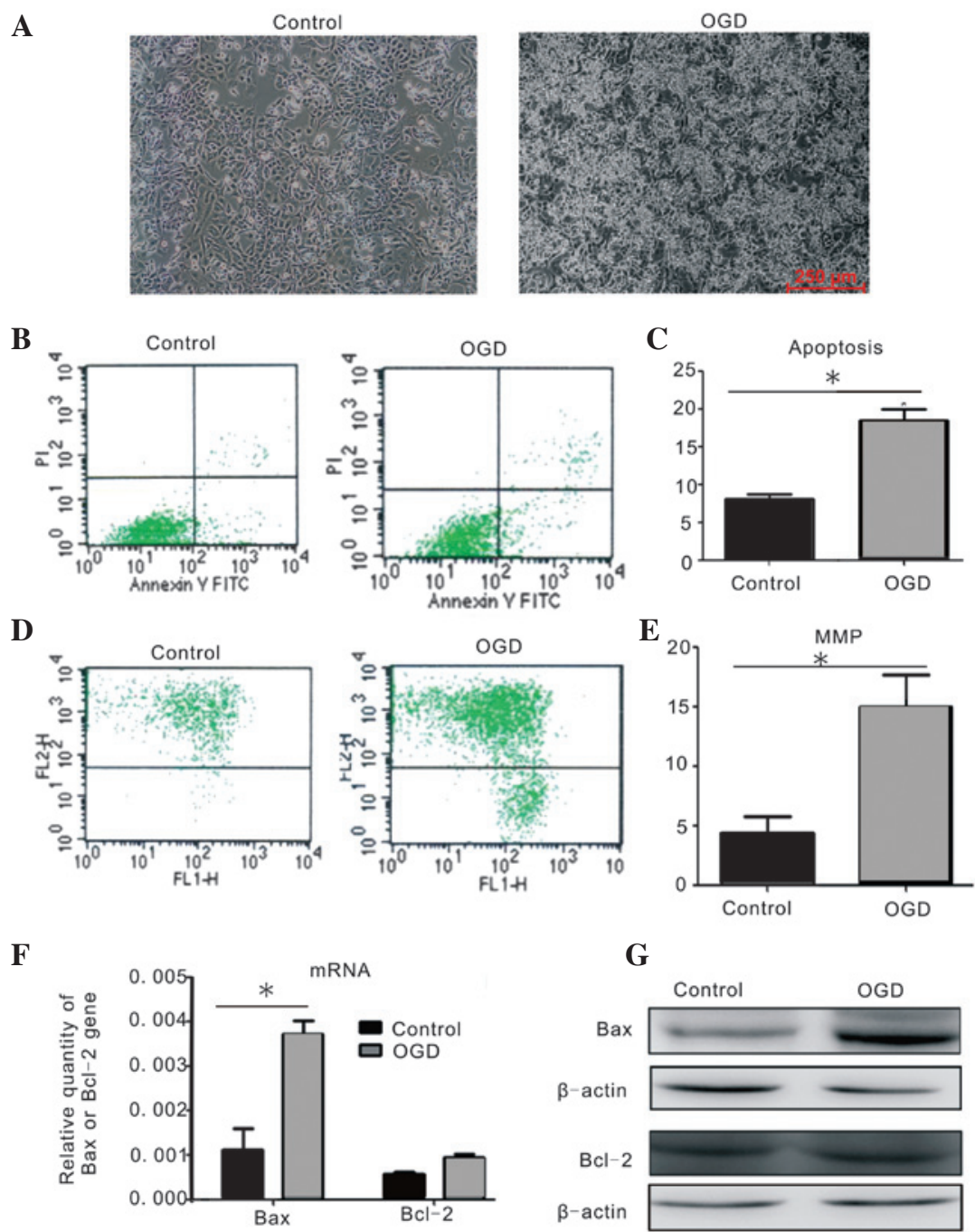

Figure 1. Apoptosis in PC12 cells injured by OGD. (A) Morphological changes in PC12 cells with or without OGD injury. Scale bar, $250 \mu \mathrm{m}$ (magnification, x 100). (B) The percentage of apoptosis in injured PC12 cells following OGD treatment as measured by flow cytometry. (C) Quantitative comparison of the percentage of apoptotic cells in the control and OGD-injured PC12 cells ( ${ }^{*} \mathrm{P}<0.05$ compared with control group, by one-way ANOVA). (D) Percentage of green fluorescent intensity of JC-1 in the PC12 cells using a mitochondrial membrane potential assay kit and flow cytometric analysis. (E) Quantitative comparison of the percentage of green fluorescence intensity in the PC12 cells with or without OGD treatment ("P $<0.05$ compared with control group, by one-way ANOVA). (F-G) Changes in mRNA and protein expression of Bax and Bcl-2 in PC12 cells following OGD injury as measured using quantitative polymerase chain reaction and western blott analysis. These results were confirmed in at least three independent experiments and normalized to $\beta$-actin (" $\mathrm{P}<0.05 \mathrm{vs}$. control, by one-way ANOVA). OGD, oxygen glucose deprivation; MMP, mitochondrial membrane potential; ANOVA, analysis of variance; Bcl-2, B-cell lymphoma 2; Bax, Bcl-2-associated X.

Measurement of apoptosis. The apoptotic rate of the PC12 cells was determined using an Annexin V-enhanced green fluorescent protein (EGFP)/propidium iodide (PI) Apoptosis Detection kit (KGA103; KeyGen Biotech, Nanjing, China) and flow cytometric analysis. Following OGD treatment, the cells were collected, washed twice with cold PBS and resuspended in $400 \mu \mathrm{l}$ of $1 \mathrm{X}$ Annexin $\mathrm{V}$ binding buffer at a concentration of $\sim 1 \times 10^{6}$ cells $/ \mathrm{ml}$. A total of $5 \mu \mathrm{l}$ of Annexin V-EGFP solution was added to the cell suspension and incubated for $15 \mathrm{~min}$ at $2-8^{\circ} \mathrm{C}$ in the dark. Next, $10 \mu 1$ of PI was added to the mixture for $5 \mathrm{~min}$. The stained cells were analyzed using flow cytometry (FacsCalibur; BD Biosciences, Franklin Lakes, NJ, USA) within 1 h. Annexin V labeled with a fluorophore identified cells at the early stage of apoptosis, and PI, a fluorescent nucleic acid binding dye, stained cells in the middle and late stages of apoptosis. The apoptotic rate was calculated as the percentage of Annexin
V-positive and PI-negative cells divided by the total number of cells in the imaged region.

Measurement of the mitochondrial membrane potential (MMP). The MMP change of the PC12 cells was measured using a mitochondrial membrane potential assay kit with JC-1 (C2006; Beyotime Institute of Biotechnology, Shanghai, China) and flow cytometric analysis. JC-1 is widely used as a fluorescent probe to detect the MMP. When the MMP is high, the dye assembles in a dimeric configuration in the mitochondrial matrix and emits a red fluorescent signal. When the MMP is low, the dye exists in a monomeric configuration and emits a green fluorescent signal. Therefore, changes in the different fluorescent signals emitted by JC-1 reflect changes in the MMP. The percentage of fluorescent intensity reflects changes in the MMP and the increase in the percentage of green fluorescence intensity suggests a decrease in the MMP. A decrease in the MMP is an early indicator of 
apoptosis. The MMP measurement was performed according to the manufacturer's instructions. Briefly, 1-6x10 5 cells were incubated in a mixture of $0.5 \mathrm{ml}$ culture medium and $0.5 \mathrm{ml}$ JC-1 staining working fluid at $37^{\circ} \mathrm{C}$ for $20 \mathrm{~min}$. Following centrifugation, the cells were washed twice with cold JC-1 staining buffer (1x) and resuspended using JC-1 staining buffer for analysis via flow cytometry within $1 \mathrm{~h}$.

Statistical analysis. Values are expressed as the mean \pm standard error of measurements. Significant differences between samples were analyzed using a one-way analysis of variance (ANOVA), a repeated-measures ANOVA and the Student-Newman-Keuls test (SNK-q). All statistical analyses were computed using GraphPad Prism 5.0 (GraphPad Software, Inc., La Jolla, CA, USA) software by professional staff. $\mathrm{P} \leq 0.05$ was considered to indicate a statistically significant difference.

\section{Results}

Construction of an OGD model in PC12 cells. The PC12 cell line was derived from transplantable rat pheochromocytoma. The PC12 cells in culture exhibited clearly visible spindle-or oval-shaped cell bodies, while they exhibited shrinkage, light refraction with decreased, chrysanthemum-like cell body shrinkage and an increase in floating cells following injury with OGD when compared with the normal PC12 cells (Fig. 1A). Flow cytometric analysis indicated that the apoptotic rate of PC12 cells following OGD treatment increased to $18.51 \pm 1.42 \%$, which was significantly different from that in the uninjured group $(8.08 \pm 0.90 \%$; Fig. 1B and C). Flow cytometry was also used to analyze the changes in the MMP in the PC12 cells. It was identified that the percentage of green fluorescence intensity of JC-1 in PC12 cells also significantly increased, from $4.14 \pm 1.47 \%$ in the untreated group to $14.47 \pm 3.73 \%$ in the OGD-injured group (Fig. 1D and E), suggesting that OGD injury decreased the MMP in PC12 cells. The mRNA and protein expression of apoptosis-associated-factor Bax was upregulated following ODG injury. The mRNA expression of anti-apoptotic factor Bcl-2 in the OGD-injured group was marginally increased when compared with the uninjured group (Fig. 1F); however, the protein expression of Bcl-2 was not significantly different (Fig. 1G). These data demonstrated that the OGD model in vitro effectively induced apoptosis of PC12 cells.

Effect of ATRA treatment on PC12 cells injured by OGD. To examine the effect of ATRA, the PC12 cells were pretreated with four different concentrations prior to OGD injury. As revealed in Fig. 2A, the apoptotic rate of PC12 cells following OGD was $14.01 \pm 1.26$ and $11.48 \pm 1.53 \%$ in the 2 and $4 \mu \mathrm{mol} / 1$ ATRA groups, respectively, which was significantly decreased compared with the OGD-treated group $(\mathrm{P}<0.05)$. However, the apoptotic rate of the $0.5 \mu \mathrm{mol} / 1$ ATRA-treated group was equal to that of the OGD-treated group, whereas the ratio was significantly increased following $20 \mu \mathrm{mol} / 1$ ATRA treatment $(\mathrm{P}<0.05)$. The percentage of green fluorescent intensity reversely indicating MMP levels was in parallel with the changes in apoptotic rates (Fig. 2B). Pretreatment with $4 \mu \mathrm{mol} / \mathrm{l}$ ATRA increased the MMP
A

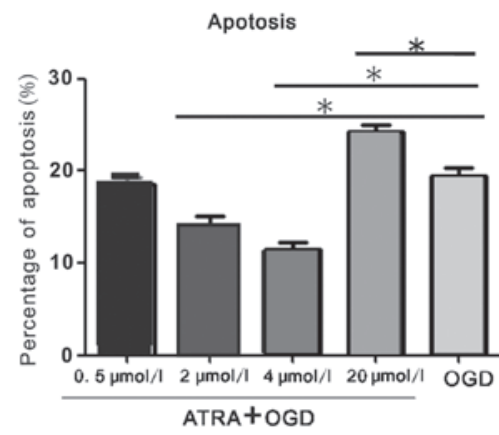

$\mathbf{B}$

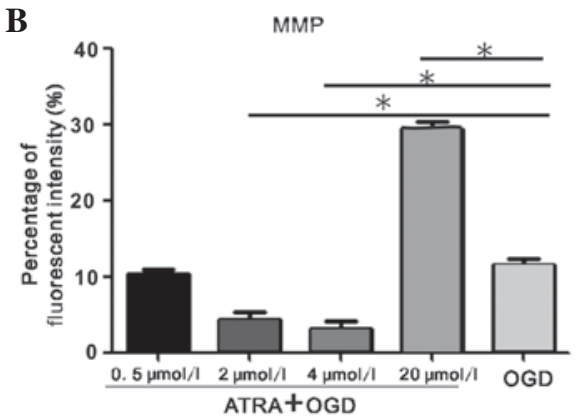

Figure 2. Detection of apoptosis and MMP change in PC12 cells injured by OGD following ATRA treatment. (A) Quantification of the apoptotic rate in OGD-injured PC12 cells following treatment with ATRA at four concentrations $(0.5,2,4$ and $20 \mu \mathrm{mol} / \mathrm{l})$ as measured by FACS ( $\mathrm{P}<0.05$ vs. the OGD group without ATRA treatment, by one-way ANOVA). (B) Quantification of the percentage of green fluorescence intensity reversely correlated with the MMP in OGD-injured PC12 cells following ATRA treatment at four concentrations $(0.5,2,4$ and $20 \mu \mathrm{mol} / 1)$ as measured by FACS ( $(\mathrm{P}<0.05$ vs. the OGD group without ATRA treatment, by one-way ANOVA). The above data were obtained from at least three independent experiments and the reported values are expressed as the mean \pm standard error of the mean. MMP, mitochondrial membrane potential; OGD, oxygen glucose deprivation; ATRA, all-transretinoic acid; ANOVA, analysis of variance; FACS, fluorescence-activated cell sorting.

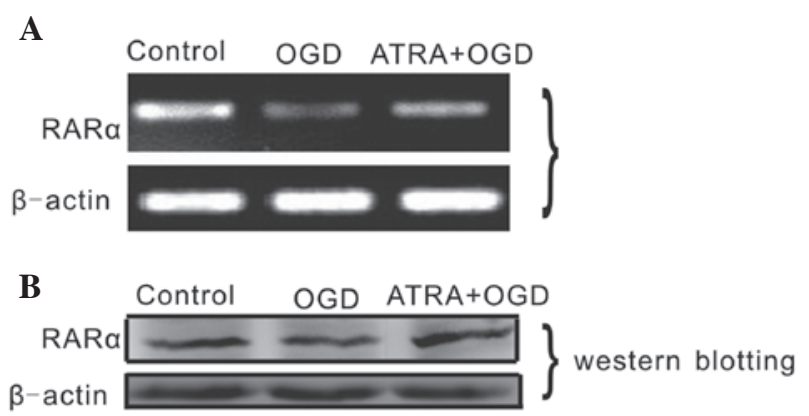

Figure 3. RAR $\alpha$ mRNA and protein expression is altered following OGD treatment with ATRA at $4 \mu \mathrm{mol} / 1$. (A) Changes in RAR $\alpha$ mRNA expression levels in the PC12 cells among the three groups, including the control without OGD and ATRA treatments, OGD alone and OGD treated with ATRA. (B) Changes in RAR $\alpha$ protein expression in the PC12 cells following various treatments, including the control without OGD and ATRA treatments, OGD alone and OGD treated by ATRA. The above data were confirmed in at least three independent experiments. RAR $\alpha$, retinoic acid receptor $\alpha$; OGD, oxygen glucose deprivation; ATRA, all-trans-retinoic acid.

and decreased the apoptotic rate to a greater extent than 2 $\mu \mathrm{mol} / \mathrm{l}$ ATRA; however, the differences were not statistically significant. The $4 \mu \mathrm{mol} / \mathrm{l}$ ATRA treatment was determined to be the optimal anti-apoptotic concentration of ATRA to use 
A

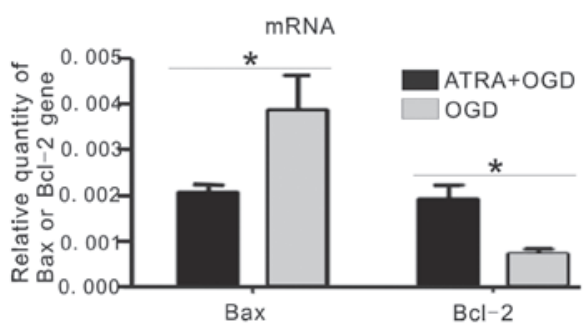

B

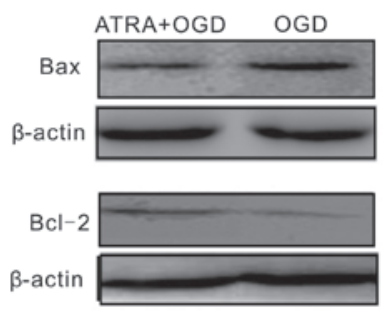

Figure 4. ATRA at $4 \mu \mathrm{mol} / 1$ affects the expression levels of Bax and Bcl-2 in PC12 cells damaged by OGD. (A) Transcriptional expression of Bax and Bcl-2 in PC12 cells following OGD injury with $4 \mu \mathrm{mol} / 1$ ATRA treatment. The mRNA expression was measured by quantitative polymerase chain reaction and the results were confirmed in at least three batches of independent experiments using $\beta$-actin normalization (" $\mathrm{P}<0.05$ vs. the OGD group without ATRA treatment, by one-way analysis of variance). (B) Protein expression of Bax and Bcl-2 in the OGD-injured PC12 cells following $4 \mu$ mol/1 ATRA treatment was analyzed by western blot analysis. The samples were equally loaded and normalized to $\beta$-actin in each lane. OGD, oxygen glucose deprivation; ATRA, all-trans-retinoic acid; Bcl-2, B-cell lymphoma 2; Bax, Bcl-2-associated X.

A

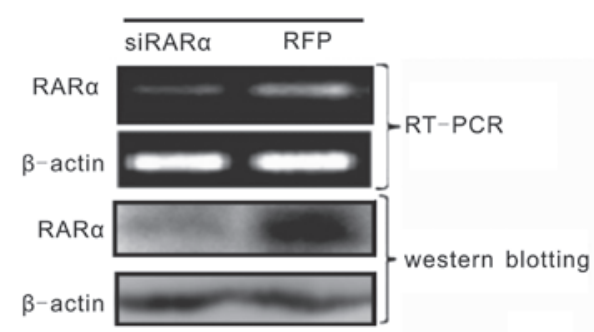

D

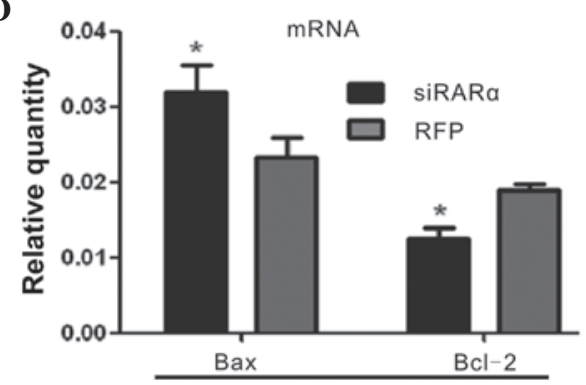

B

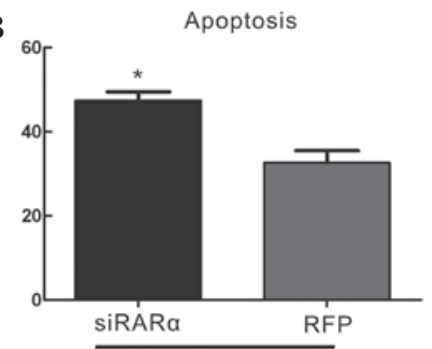

$\mathbf{E}$

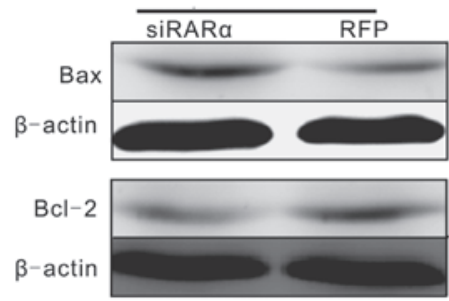

C

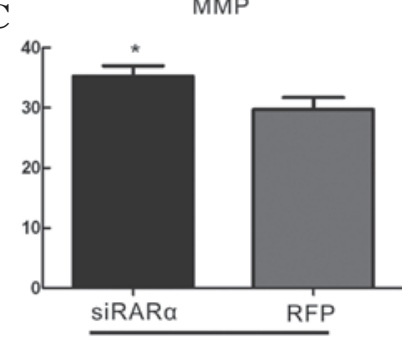

Figure 5. Ad-siRAR $\alpha$ blocks the anti-apoptotic effects of $4 \mu \mathrm{mol} / 1$ ATRA on PC12 cells treated by OGD. (A) Ad-siRAR $\alpha$ significantly inhibited mRNA and protein expression of RAR $\alpha$ in PC12 cells treated by OGD and $4 \mu \mathrm{mol} / 1$ ATRA. (B) Quantitative comparison of the percentage of apoptosis in OGD+ATRA-treated PC12 cells following transfection with either Ad-siRAR $\alpha$ or RFP. Fluorescence-activated cell sorting results were confirmed in at least three independent experiments. Values are expressed as the mean \pm SEM ("P<0.05 vs. the RFP+ATRA+OGD group, by one-way ANOVA). (C) Quantification of the percentage of green fluorescence intensity of JC-1 reversely correlated with the MMP in the OGD+ATRA-treated PC12 cells following transfection with either Ad-siRAR $\alpha$ or RFP. These tests were performed in at least three independent experiments. Values are expressed as the mean $\pm \mathrm{SEM}\left({ }^{*} \mathrm{P}<0.05\right.$ vs. the RFP+ATRA+OGD group, by one-way ANOVA). (D-E) Changes in the expression levels of Bax and Bcl-2 following Ad-siRAR $\alpha$ transfection of the OGD+ATRA-treated PC12 cells, as measured by quantitative polymerase chain reaction and western blot analysis. Data from at least three independent experiments were analyzed and normalized to $\beta$-actin. Values are expressed as the mean $\pm \mathrm{SEM}\left({ }^{*} \mathrm{P}<0.05\right.$ vs. the RFP+ATRA+OGD group, by one-way ANOVA). RAR $\alpha$, retinoic acid receptor $\alpha$; OGD, oxygen glucose deprivation; ATRA, all-trans-retinoic acid; RFP, red fluorescent protein; MMP, mitochondrial membrane potential; SEM, standard error of the mean; ANOVA, analysis of variance; Bcl-2, B-cell lymphoma 2; Bax, Bcl-2-associated X; siRNA, small interfering RNA; Ad, adenovirus.

in the subsequent experiments, as it caused apoptosis more effectively.

RAR $\alpha$ mRNA and protein expression levels are altered following OGD treatment with ATRA at $4 \mu \mathrm{mol} / \mathrm{l}$. To examine the effects of ATRA on PC12 cells, the changes in RAR $\alpha$ expression levels were detected by qPCR and western blot analysis (Fig. 3). The mRNA expression of RAR $\alpha$ in OGD-injured PC12 cells increased following treatment with $4 \mu \mathrm{mol} / 1$ ATRA, whereas the expression decreased following OGD injury without any other treatment. The protein expression levels of RAR $\alpha$ in OGD-injured PC12 cells treated with $4 \mu \mathrm{mol} / 1$ ATRA were higher than those in OGD-injured cells without ATRA treatment. This finding suggested that the levels of RAR $\alpha$ expression are affected by OGD and $4 \mu \mathrm{mol} / 1$ ATRA, and that RAR $\alpha$ is involved in ATRA-mediated signaling during OGD.

Anti-apoptotic effect of $4 \mu \mathrm{mol} / \mathrm{l}$ ATRA. To examine the anti-apoptotic effect of $4 \mu \mathrm{mol} / 1$ ATRA, the expression of apoptosis-associated factors was examined. As revealed in Fig. 4A, when compared with the OGD-injured cells without ATRA treatment, the mRNA expression of Bax was significantly reduced by $4 \mu \mathrm{mol} / \mathrm{l}$ ATRA treatment following OGD injury $(\mathrm{P}<0.05)$. Furthermore, the mRNA expression of $\mathrm{Bcl}-2$ was significantly increased in the $4 \mu \mathrm{mol} / \mathrm{l}$ ATRA+OGD 
group $(\mathrm{P}<0.05)$. The changes in the expression levels of Bax and $\mathrm{Bcl}-2$ were consistent with the changes in their protein expression, as detected by western blot analysis (Fig. 4B). These results suggested that a $4 \mu \mathrm{mol} / 1$ ATRA pre-treatment for $24 \mathrm{~h}$ may protect the $\mathrm{PC} 12$ cells from the induction of apoptosis caused by OGD.

$R A R \alpha$ regulates the anti-apoptotic effect of ATRA treatment on PC12 cells injured by $O G D$. The expression levels of RAR $\alpha$ were altered by ATRA in PC12 cells following OGD injury. To investigate whether RAR $\alpha$ regulates the anti-apoptotic effect of ATRA treatment, the OGD-injured PC12 cells were infected with Ad-siRAR $\alpha$. The mRNA and protein expression levels of RAR $\alpha$ were inhibited in the PC12 cells with OGD+ATRA treatment by Ad-siRAR $\alpha$ when compared with those in cells transfected with the Ad-RFP control vector (Fig. 5A). Following transfection with Ad-siRAR $\alpha$, the percentages of apoptotic cells and the green fluorescence intensity reversely correlated with the MMP were significantly higher than those of the Ad-RFP group following $4 \mu \mathrm{mol} / \mathrm{l} \mathrm{ATRA+OGD} \mathrm{treatment} \mathrm{(Fig.} \mathrm{5B} \mathrm{and}$ C). Furthermore, the PC12 cells transfected with Ad-siRARa had increased mRNA expression levels of Bax and decreased mRNA expression levels of Bcl-2 following the $4 \mu \mathrm{mol} / 1$ ATRA+OGD treatment (Fig. 5D). The changes in Bax and Bcl-2 protein expression were similar to the changes in mRNA expression in the PC12 cells transfected with Ad-siRAR $\alpha$ and treated with $4 \mu \mathrm{mol} / 1$ ATRA+OGD (Fig. 5E). These data indicated that pre-treatment with $4 \mu \mathrm{mol} / \mathrm{l}$ ATRA suppressed apoptosis in OGD-injured PC12 cells through the RAR $\alpha$ signaling pathway.

\section{Discussion}

Vitamin A is an essential fat-soluble micronutrient that contributes to development and growth. RA, which includes ATRA, 13-cis-retinoic acid (13-cis-RA) and 9-cis-retinoic acid (9-cis-RA), is a main active metabolite of VA in vivo. ATRA can regulate the transcriptional activity of downstream genes through retinoic acid nuclear receptor-mediated signal transduction. VA deficiency during pregnancy downregulates the expression of RAR $\alpha$ and NR1 through $\mathrm{Ca}^{2+}$ signaling and decreases the learning and memory function of offspring (4). Jacobs et al (15) found that RA promotes neurogenesis and that RA deficiency leads to lower survival of nerve cells in vivo. RA also affects the proliferation and apoptosis of damaged nerve cells (16). Shinozaki et al (17) found that precursor nerve cells differentially weakened in the hippocampal dentate gyrus granule cell layer if RA was deprived in adult rats. However, the regulatory effects of VA on the anti-apoptotic mechanisms of neuron cells remain elusive.

In the present study, an in vitro OGD model in PC12 cells was successfully established, which exhibited an increase in the percentage of apoptosis and a decrease in the levels of MMP, but also significantly enhanced levels of Bax expression. These results demonstrated that OGD damage leads to acute apoptosis in PC12 cells. Furthermore, it was identified that 2 and $4 \mu \mathrm{mol} / 1$ ATRA significantly suppressed the apoptosis of PC12 cells injured by OGD and effectively maintained MMP stability. In addition, $4 \mu \mathrm{mol} / 1$ ATRA upregulated Bcl-2 expression and downregulated Bax expression. However, $0.5 \mu \mathrm{mol} / 1$ ATRA was not able to reverse the apoptosis that occurred in
OGD-injured PC12 cells due to its low concentration, and $20 \mu \mathrm{mol} / 1$ ATRA produced increased levels of apoptosis in PC12 cells due to toxicity at such a high concentration. These results indicated that ATRA had different biological effects at different concentrations. Numerous studies have demonstrated that ATRA is correlated with apoptosis (18-21). In the present study, $4 \mu \mathrm{mol} / \mathrm{l}$ ATRA treatment more effectively suppressed apoptosis in the PC12 cells following OGD treatment and it was optimal to further reveal the mechanism of ATRA regulation.

ATRA receptors include two types, RARs and RXRs, which both contain three subtypes $(\alpha, \beta$ and $\gamma)$. Most commonly, these receptors form homo- or heterodimers that combine with region-specific RARs elements (RARE) in the promoter region of a target gene to regulate its transcription. RAR $\alpha$ is a main receptor in hippocampal development in the rat, particularly in learning and memory function. A previous in vivo study by our group demonstrated that VA levels affect learning and memory function via the RAR $\alpha$ pathway (22). In the present study, OGD injury decreased the levels of RAR $\alpha$ expression in PC12 cells, and the expression of RAR $\alpha$ increased when ATRA was added to PC12 cells following OGD treatment, suggesting that the ATRA signaling pathway may regulate apoptosis following OGD damage. Katsuki et al (23) demonstrated that ATRA upregulates RAR $\alpha$ expression and enhances BDNF reactivity to protect the dopaminergic neural system from damage.

To confirm a regulatory role of RAR $\alpha$, an Ad-siRAR $\alpha$ recombinant adenovirus was used. It was revealed that Ad-siRAR $\alpha$ significantly blocked RAR $\alpha$ expression in PC12 cells following ATRA and OGD treatment. In PC12 cells subjected to ATRA and OGD treatment, inhibition of RAR $\alpha$ significantly increased the percentage of apoptosis, decreased the MMP, and also upregulated Bax expression and downregulated $\mathrm{Bcl}-2$ expression. These findings demonstrated that the anti-apoptotic properties of ATRA may be regulated through the RAR $\alpha$ signaling pathway. Therefore, future studies will focus on the regulatory effect of RAR $\alpha$ on the anti-apoptotic Bcl-2 gene and the pro-apoptotic Bax gene.

In conclusion, an in vitro OGD model in PC12 cells was successfully established in the present study. Pretreatment with ATRA at a concentration of $2-4 \mu \mathrm{mol} / 1$ had an anti-apoptotic effect on OGD-injured PC12 cells via the RAR $\alpha$ signaling pathway, suggesting a new method of adjuvant therapy for patients with brain damage.

\section{Acknowledgements}

This study was supported by grants from the National Natural Science Foundation of China (grant nos. 30830106 and 81100454 to Professor Tingyu $\mathrm{Li}$ and nos. 30872670 and 81271385 to Dr Jie Chen) and the Project of Chongqing Municipal Health Bureau, China (no. 2009-1-41 to Dr Jie Chen).

\section{References}

1. Janesick A, Shiotsugu J, Taketani M and Blumberg B: RIPPLY3 is a retinoic acid-inducible repressor required for setting the borders of the pre-placodal ectoderm. Development 139: 1213-1224, 2012. 
2. Hale LA, Tallafuss A, Yan YL, Dudley L, Eisen JS and Postlethwait JH: Characterization of the retinoic acid receptor genes raraa, rarab and rarg during zebrafish development. Gene Expr Patterns 6: 546-555, 2006.

3. Grandel H, Lun K, Rauch GJ, et al: Retinoic acid signalling in the zebrafish embryo is necessary during pre-segmentation stages to pattern the anterior-posterior axis of the CNS and to induce a pectoral fin bud. Development 129: 2851-2865, 2002.

4. Zhang X, Chen K, Chen J, Liu YX, Qu P and Li TY: Effect of marginal vitamin A deficiency during pregnancy on retinoic acid receptors and $\mathrm{N}$-methyl-D-aspartate receptor expression in the offspring of rats. J Nutr Biochem 22: 1112-1120, 2011.

5. Jiang W, Yu Q, Gong M, et al: Vitamin A deficiency impairs postnatal cognitive function via inhibition of neuronal calcium excitability in hippocampus. J Neurochem 121: 932-943, 2012.

6. Jiang T, Gao L, Shi J, Lu J, Wang $Y$ and Zhang $Y$ : Angiotensin-(1-7) modulates renin-angiotensin system associated with reducing oxidative stress and attenuating neuronal apoptosis in the brain of hypertensive rats. Pharmacol Res 67: 84-93, 2013.

7. Raveendran AT and Skaria PC: Learning and cognitive deficits in hypoxic neonatal rats intensified by BAX mediated apoptosis: protective role of glucose, oxygen, and epinephrine. Int J Neurosci 123: 80-88, 2013.

8. Cheng O, Li Z, Han Y, Jiang Q, Yan Y and Cheng K: Baicalin improved the spatial learning ability of global ischemia/reperfusion rats by reducing hippocampal apoptosis Brain Res 1470: 111-118, 2012.

9. Wang YQ, Wang L, Zhang MY, et al: Necrostatin-1 suppresses autophagy and apoptosis in mice traumatic brain injury model Neurochem Res 37: 1849-1858, 2012.

10. Hill JW, Poddar R, Thompson JF, Rosenberg GA and Yang Y: Intranuclear matrix metalloproteinases promote DNA damage and apoptosis induced by oxygen-glucose deprivation in neurons. Neuroscience 220: 277-290, 2012.

11. Piao CS, Loane DJ, Stoica BA, et al: Combined inhibition of cell death induced by apoptosis inducing factor and caspases provides additive neuroprotection in experimental traumatic brain injury. Neurobiol Dis 46: 745-758, 2012.

12. Chaung WW, Wu R, Ji Y, et al: Peripheral administration of human adrenomedullin and its binding protein attenuates stroke-induced apoptosis and brain injury in rats. Mol Med 17: 1075-1083, 2011
13. Tang LH, Xia ZY, Zhao B, Wei XD, Luo T and Meng QT: Phosphocreatine preconditioning attenuates apoptosis in ischemia-reperfusion injury of rat brain. J Biomed Biotechnol 2011: 107091, 2011

14. Jin W, Ni H, Dai Y, et al: Effects of tert-butylhydroquinone on intestinal inflammatory response and apoptosis following traumatic brain injury in mice. Mediators Inflamm 2010: 502564, 2010.

15. Jacobs S, Lie DC, DeCicco KL, et al: Retinoic acid is required early during adult neurogenesis in the dentate gyrus. Proc Natl Acad Sci USA 103: 3902-3907, 2006.

16. Maden M: Retinoic acid in the development, regeneration and maintenance of the nervous system. Nat Rev Neurosci 8: 755-765, 2007.

17. Shinozaki Y, Sato Y, Koizumi S, Ohno Y, Nagao T and Inoue K: Retinoic acids acting through retinoid receptors protect hippocampal neurons from oxygen-glucose deprivation-mediated cell death by inhibition of c-jun-N-terminal kinase and p38 mitogen-activated protein kinase. Neuroscience 147: 153-163, 2007.

18. Karmakar S, Banik NL and Ray SK: Combination of all-trans retinoic acid and paclitaxel-induced differentiation and apoptosis in human glioblastoma U87MG xenografts in nude mice. Cancer 112: 596-607, 2008

19. Keedwell RG, Zhao Y, Hammond LA, et al: A retinoid-related molecule that does not bind to classical retinoid receptors potently induces apoptosis in human prostate cancer cells through rapid caspase activation. Cancer Res 64: 3302-3312, 2004.

20. Thin TH, Li L, Chung TK, Sun H and Taneja R: Stra13 is induced by genotoxic stress and regulates ionizing-radiation-induced apoptosis. EMBO Rep 8: 401-407, 2007.

21. Li J, Orr B, White K, et al: Chmp 1A is a mediator of the anti-proliferative effects of all-trans retinoic acid in human pancreatic cancer cells. Mol Cancer 8: 7, 2009.

22. Jiang W, Wen EY, Gong M, et al: The pattern of retinoic acid receptor expression and subcellular, anatomic and functional area translocation during the postnatal development of the rat cerebral cortex and white matter. Brain Res 1382: 77-87, 2011.

23. Katsuki H, Kurimoto E, Takemori S, et al: Retinoic acid receptor stimulation protects midbrain dopaminergic neurons from inflammatory degeneration via BDNF-mediated signaling. J Neurochem 110: 707-718, 2009. 